\title{
Bevacizumab combined with low-dose $S-1$ as maintenance therapy with a long progression-free survival in an elderly patient with heavily pre-treated advanced gastric cancer: A case report
}

\author{
JUEMIN FANG, HUI WANG and QING XU \\ Department of Medical Oncology, Shanghai Tenth People's Hospital, \\ Tongji University School of Medicine, Shanghai 200072, P.R. China
}

Received August 26, 2012; Accepted October 10, 2012

DOI: $10.3892 /$ br.2012.37

\begin{abstract}
Gastric cancer is known to have a high incidence in several countries around the world, while the treatment of advanced gastric cancer remains a challenge. Recent studies have shown that the antibody bevacizumab, a monoclonal vascular endothelial growth factor antibody, is effective in several solid tumors, while experience regarding its effect on gastric cancer remains limited. An 84-year-old patient with advanced gastric remnant cancer with liver and retroperitoneal lymph node metastasis treated in our hospital benefitted from the use of bevacizumab. Since previous treatment with multiple chemotherapeutic agents resulted in progressive disease (PD), a combined treatment with bevacizumab (intravenously) and low-dose S-1 (orally) was administered. With this individualized treatment, the patient exhibited stable disease (SD) and therapy was maintained for a long period of time as maintenance therapy, with a progression-free survival of 25 months prior to PD. The serum tumor marker cancer antigen (CA) 199 decreased from 508.7 to $188.1 \mathrm{ng} / \mathrm{ml}$. No severe side-effects of bevacizumab were observed, with the exception of controlled grade I bleeding gums due to the long-term use of bevacizumab. Although no large-scale clinical trials have been conducted to evaluate the role of bevacizumab in maintenance therapy and second- or even third-line treatment of advanced gastric cancer, we showed that bevacizumab combined with S-1 was effective and well-tolerated by this patient, suggesting that it be considered a viable option for elderly patients with advanced gastric cancer as maintenance therapy and that it provide a novel treatment for advanced gastric cancer. However, additional clinical trials are required to evaluate the
\end{abstract}

Correspondence to: Dr Qing Xu, Department of Medical Oncology, Tenth People's Hospital of Tongji University, Tongji University, 301 Yanchang Road, Shanghai 200072, P.R. China

E-mail: xuqingmd@yahoo.com.cn

Key words: bevacizumab, S-1, gastric cancer, maintenance therapy exact effects of long-term bevacizumab treatment on patients with advanced gastric cancer.

\section{Introduction}

Gastric cancer remains a worldwide health problem. In the USA, the number of new cases and deaths due to gastric cancer in 2012 is estimated to be 21,320 and 10,540, respectively (1). At an international level, most cases are diagnosed in China. Gastric cancer is often diagnosed at an advanced stage. Effective chemotherapeutic agents for advanced gastric cancer, including fluorouracil, etoposide, cisplatin and irinotecan, are limited and are severely toxic, thus alternative salvage therapies are needed $(2,3)$.

Recently, the use of the vascular endothelial growth factor (VEGF) antibody has been proven to be effective in metastatic solid tumors, such as ovarian and colorectal cancers $(4,5)$. In gastric cancer, a multinational, randomized, placebo-controlled phase III study (AVAGAST) demonstrated that adding bevacizumab to capecitabine-cisplatin in the first-line treatment of advanced gastric cancer has the potential to improve progression-free survival (PFS) and the overall response (OR) rate (6). Several ongoing phase II clinical trials also suggest that better outcomes are observed after bevacizumab treatment. Moreover, a phase III MACRO study suggested that maintenance therapy with the single-agent bevacizumab may be an appropriate option in patients with metastatic colorectal cancer (mCRC), after induction XELOX plus bevacizumab (4). However, the information available on the efficacy of bevacizumab for the treatment of advanced and metastatic gastric cancer is limited, compared to colorectal and ovarian cancers.

$\mathrm{S}-1$ is a novel oral fluorouracil analogue. A phase III clinical trial confirmed that S-1 was effective and safe in advanced gastric cancer as a single-agent treatment or combined with cisplatin (7). Recently, a phase II study of S-1 as first-line treatment for elderly patients with advanced gastric cancer suggested that S-1 monotherapy was safe and useful for the elderly ( $>75$ years old), when the dose was carefully selected (8). In this case report, an 84 year-old patient, who underwent treatment with multiple chemotherapeutic agents, was administered combined treatment with bevacizumab (intravenously) 


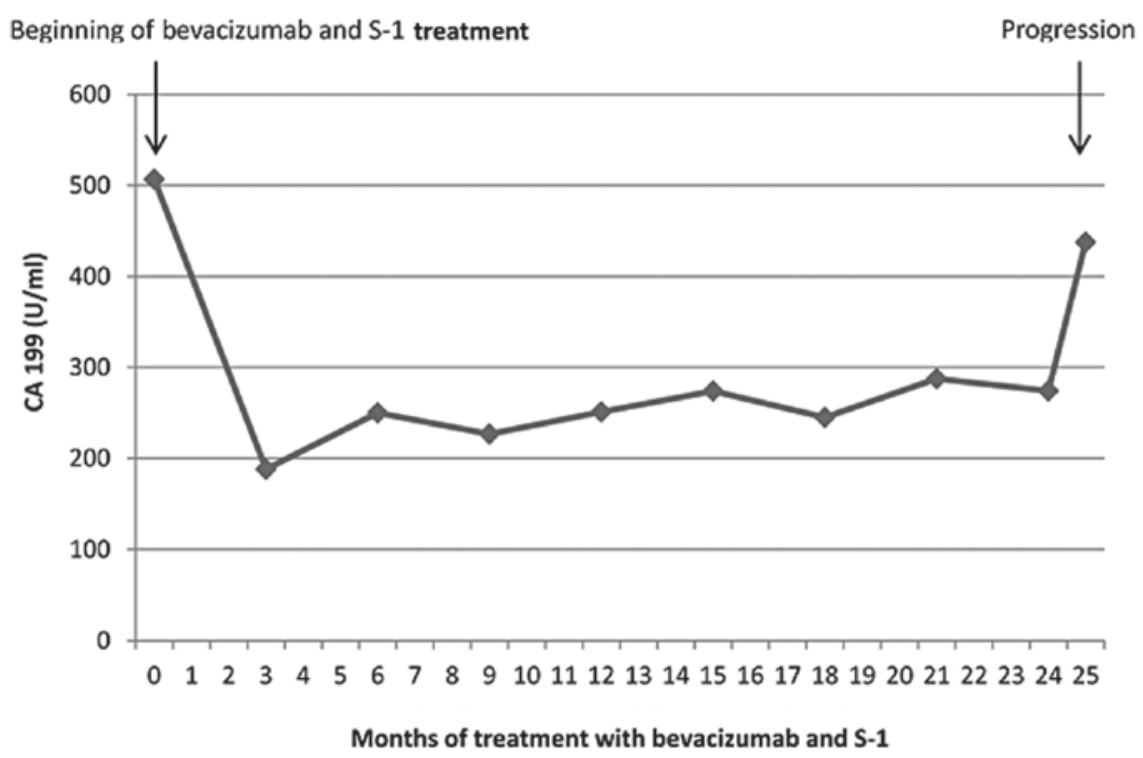

Figure 1. Course of cancer antigen 199 (CA 199) from May 2008 to July 2010 is shown.

with low-dose S-1 (orally), which was more convenient and less toxic.

\section{Case report}

We report the case of an 84-year-old male individual, hospitalized in January, 2008 with advanced gastric remnant cancer with liver and retroperitoneal lymph node metastasis.

In 2003, the patient underwent subtotal gastrectomy when primarily diagnosed with gastric malignant tumor, confirmed by pathological analysis. No additional adjuvant treatment was administered following surgery. After a 3-year follow-up, the patient was diagnosed with liver metastasis by abdominal B-mode ultrasound in October, 2006. Subsequently, abdominal computed tomography (CT) and whole-body positron emission tomography (PET)-CT also showed liver and retroperitoneal lymph node metastasis. Gastric remnant cancer was confirmed by gastroscopy and adenocarcinoma was histopathologically verified. Due to dissemination of the disease and the age of the patient, radical surgery was inapplicable and palliative chemotherapy was administered comprising oxaliplatin $\left(130 \mathrm{mg} / \mathrm{m}^{2}\right.$ day 1) and xeloda (1,000 mg/m² days 1-14) q21 for 6 cycles between October, 2006 and March, 2007 with stable disease (SD). In December, 2007, the abdominal CT scan showed progressive disease (PD), according to Response Evaluation Criteria In Solid Tumors (RECIST) criteria. Therefore, the chemotherapeutic regimen was changed to docetaxel, cisplatin and 5-fluorouracil (DCF) including docetaxel ( $75 \mathrm{mg} / \mathrm{m}^{2}$ day 1), cisplatin $\left(75 \mathrm{mg} / \mathrm{m}^{2}\right.$ days $\left.1-3\right)$ and 5 -fluorouracil $(750 \mathrm{mg}$ / $\mathrm{m}^{2}$ days 1-5), lasting for 4 cycles between January, 2008 and March, 2008 until PD was confirmed by abdominal CT.

Considering the age of the patient and the administration of multiple chemotherapeutic agents with disease progression, bevacizumab $(7.5 \mathrm{mg} / \mathrm{kg})$ was administered every 3 weeks intravenously starting in May, 2008. Treatment was well-tolerated after three cycles of bevacizumab, when low-dose S-1 (1\# bid days 1-14 q28; orally) administration was added to enhance treatment efficacy. The administration of bevacizumab and S-1 led to a PFS of 25 months with cancer antigen (CA) 199 decreasing from 508.7 to $188.1 \mathrm{ng} / \mathrm{ml}$ (Fig. 1). No significant side-effects of bevacizumab were observed, with the exception of the controlled grade I bleeding gums. Grade II skin rash of the upper limbs by $\mathrm{S}-1$ as per the National Cancer Institute-Common Toxicity Criteria (NCI-CTC) was alleviated following steroid use.

In June, 2010, imaging demonstrated progression of the disease, thus, S-1 was replaced and the patient received 4 cycles of bevacizumab $(7.5 \mathrm{mg} / \mathrm{kg})$, liposomal doxorubicin $\left(20 \mathrm{mg} / \mathrm{m}^{2}\right.$ day 1) and oxaliplatin $\left(100 \mathrm{mg} / \mathrm{m}^{2} \mathrm{~d}\right.$ 2) until PD. Due to non-medical reasons, the bevacizumab treatment was terminated and on the basis of the status of the patient, best support care (BSC) was provided. The patient developed brain metastasis 2 months after bevacizumab and chemotherapeutic drug treatment termination. The patient succumbed to the disease in March, 2011 due to dyscrasia.

\section{Discussion}

Triplet combination chemotherapy containing a platinumbased compound, a fluoropyrimidine with an anthracycline (frequently added in Europe) or a taxane (more often used in the USA and elsewhere) has been considered as first-line treatment for advanced gastric cancer, resulting in higher response rates and modest improvement in overall survival (OS) compared to doublet combination chemotherapies. However, no standard treatment is currently available for patients with advanced gastric cancer after failure of other treatment approaches. Additionally, it is more difficult to select treatment for elderly patients with advanced gastric cancer due to the low tolerance following the administration of multiple chemotherapeutic agents. Therefore, there is a need for alternative salvage options.

Bevacizumab has been approved for use in the treatment of colorectal and non-small cell lung cancers in several countries. 
Regarding gastric cancer, a phase II study of modified DCF treatment with bevacizumab in patients with metastatic gastroesophageal adenocarcinoma improved 6-month PFS to $79 \%$, and the median and 2-year OS were 16.8 months and $37 \%$, respectively (9). An additional phase II study of oxaliplatin, and docetaxel combined with bevacizumab showed that median PFS was 6.6 months and median survival was 11.1 months. Complete response was documented in $2 / 38$ patients $(5 \%)$ with locally advanced and metastatic gastric and gastroesophageal junction cancer, partial responses in $14 / 38(37 \%)$, and SD in $14 / 38$ patients (37\%) (10). AVAGAST, a phase III study, demonstrated that adding bevacizumab to capecitabine-cisplatin in the first-line treatment of advanced gastric cancer has the potential to improve the PFS and OR rate (6). These data suggest the efficacy and safety of bevacizumab in the treatment of advanced gastric cancer.

Recently, maintenance therapy, a novel treatment strategy aiming to strengthen the efficacy of treatment has been suggested (11). Maintenance therapy could be classified as the continuation of maintenance and switch-maintenance. Continuation maintenance is a prolongation of a part of the former chemotherapeutic agents or molecularly-targeted agent until PD, when the efficacy of former treatment is SD or above. Switch-maintenance is defined as a different cytotoxic chemotherapy or molecularly-targeted agent administered immediately after induction therapy. Findings of large-scale randomized prospective controlled studies have shown benefits with maintenance therapy in various tumors, such as non-small cell lung cancer (12). In advanced non-small cell lung cancer, maintenance therapy with bevacizumab as tolerated until progression for platinum-based chemotherapy combined with bevacizumab was recommended in the guidelines of the American Society of Clinical Oncology (ASCO) (13). The MACRO study provided us with the value of bevacizumab in maintenance therapy in mCRC, suggesting maintenance therapy with single-agent bevacizumab or bevacizumab plus XELOX, as an appropriate option (4). Although there have been no large-scale clinical trials evaluating the role of bevacizumab in maintenance therapy in advanced and metastatic gastric cancer, the long-term use of bevacizumab with the long-term PFS of the case described here, suggested that bevacizumab was an effective and safe option for maintenance chemotherapy in advanced gastric cancer.

Furthermore, rebound tumor progression after the cessation of bevacizumab therapy in other types of cancer has been previously reported (14). The patient described in this study was treated with bevacizumab for a long period of time. Subsequently, the patient developed rapid brain metastasis two months after bevacizumab and chemotherapeutic drug treatment termination. This suggests that the expression of VEGF may rebound to elevated levels and accelerate disease progression.

In their study, Koizumi et al (7) confirmed the efficacy and safety of S-1 in a phase III study. Findings of a multicenter phase III study showed cisplatin/S-1 to be as efficacious as cisplatin/infusional fluorouracil, resulting in a significantly improved safety profile (15). In a retrospective study, Seol et al (16) showed that oral fluoropyrimidines (capecitabine or S-1) and cisplatin may be used as first-line treatment of elderly patients with advanced gastric cancer (16).
Additionally, in a phase II study, Koizumi et al (8) reported that S-1 monotherapy was safe and useful for elderly patients ( $>75$ years old), when the dose was carefully selected (8). Based on these results, low-dose S-1 was adopted for the elderly patients included in this study and it was shown that S-1 is a potentially well-tolerated and effective drug. Results of the present case also suggest that in elderly patients with low tolerance, low-dose chemotherapeutic agents may be more beneficial to patients compared to aggressive regimens with severe toxicity.

To the best of our knowledge, although no large-scale clinical trials evaluating the role of bevacizumab in maintenance therapy and second- or even third-line treatment of advanced gastric cancer have been conducted, the present study has shown that bevacizumab and low-dose oral S-1 have the potential to be a well-tolerated and excellent option in elderly and heavily pretreated patients with advanced gastric cancer, when treatment choices are limited. The patient described in this case report benefitted from the individualized regimen, as indicated by the long-term PFS and good safety. Additionally, bevacizumab was demonstrated to be a viable option in maintenance chemotherapy in advanced gastric cancer. However, use of bevacizumab for the treatment of advanced gastric cancer remains to be further elucidated. Thus, future trials are required to prospectively investigate the efficacy of bevacizumab and S-1.

\section{Acknowledgements}

This study was supported by the Shanghai Science and Technology Commission (grant no. 11411950602). The authors would like to thank Juemin Fang for summarizing the case, Hui Wang for providing patient information and Qing Xu for preparing the case.

\section{References}

1. Siegel R, Naishadham D, Jemal A: Cancer statistics. CA Cancer J Clin 62: 10-29, 2012.

2. Ajani JA, Fodor MB, Tjulandin SA, et al: Phase II multi-institutional randomized trial of docetaxel plus cisplatin with or without fluorouracil in patients with untreated, advanced gastric, or gastroesophageal adenocarcinoma. J Clin Oncol 23: 5660-5667, 2005.

3. Van Cutsem E, Moiseyenko VM, Tjulandin S, et al: Phase III study of docetaxel and cisplatin plus fluorouracil compared with cisplatin and fluorouracil as first-line therapy for advanced gastric cancer: a report of the V325 Study Group. J Clin Oncol 24: 4991-4997, 2006.

4. Díaz-Rubio E, Gómez-España A, Massutí B, et al: First-line XELOX plus bevacizumab followed by XELOX plus bevacizumab or single-agent bevacizumab as maintenance therapy in patients with metastatic colorectal cancer: the phase III MACRO TTD study. Oncologist 17: 15-25, 2012.

5. Perren TJ, Swart AM, Pfisterer J, et al: A phase 3 trial of bevacizumab in ovarian cancer. N Engl J Med 365: 2484-2496, 2011.

6. Ohtsu A, Shah MA, Van Cutsem E, et al: Bevacizumab in combination with chemotherapy as first-line therapy in advanced gastric cancer: a randomized, double-blind, placebo-controlled phase III study. J Clin Oncol 29: 3968-3676, 2011.

7. Koizumi W, Narahara H, Hara T, et al: S-1 plus cisplatin versus S-1 alone for first-line treatment of advanced gastric cancer (SPIRITS trial): a phase III trial. Lancet Oncol 9: 215-221, 2008.

8. Koizumi W, Akiya T, Sato A, et al: Phase II study of S-1 as first-line treatment for elderly patients over 75 years of age with advanced gastric cancer: the Tokyo Cooperative Oncology Group study. Cancer Chemother Pharmacol 65: 1093-1099, 2010. 
9. Shah MA, Jhawer M, Ilson DH, et al: Phase II study of modified docetaxel, cisplatin, and fluorouracil with bevacizumab in patients with metastatic gastroesophageal adenocarcinoma. J Clin Oncol 29: 868-874, 2011.

10. El-Rayes BF, Zalupski M, Bekai-Saab T, et al: A phase II study of bevacizumab, oxaliplatin, and docetaxel in locally advanced and metastatic gastric and gastroesophageal junction cancers. Ann Oncol 21: 1999-2004, 2010.

11. Sibille A, Wauters I and Vansteenkiste J: Maintenance therapy for advanced non-small-cell lung cancer: ready for clinical practice? Expert Rev Anticancer Ther 12: 529-539, 2012.

12. Ahn MJ, Sun JM, Ahn JS and Park K: The effectiveness of maintenance pharmacotherapies for non-small cell lung cancer. Clin Med Insights Oncol 6: 253-262, 2012.
13. Grossi F: Management of non-small cell lung in cancer patients with stable disease. Drugs 72: 20-27, 2012.

14. Zuniga RM, Torcuator R, Jain R, et al: Rebound tumour progression after the cessation of bevacizumab therapy in patients with recurrent high-grade glioma. J Neurooncol 99: 237-242, 2010.

15. Ajani JA, Rodriguez W, Bodoky G, et al: Multicenter phase III comparison of cisplatin/S-1 with cisplatin/infusional fluorouracil in advanced gastric or gastroesophageal adenocarcinoma study: the FLAGS trial. J Clin Oncol 28: 1547-1553, 2010.

16. Seol YM, Song MK, Choi YJ, et al: Oral fluoropyrimidines (capecitabine or S-1) and cisplatin as first line treatment in elderly patients with advanced gastric cancer: a retrospective study. Jpn J Clin Oncol 39: 43-48, 2009. 